\title{
The X-ray source populations in M31 and M33
}

\author{
Wolfgang Pietsch \\ Max-Planck-Institut für extraterrestrische Physik, Giessenbachstraße, 85741 Garching, \\ Germany \\ email: wnp@mpe.mpg.de
}

\begin{abstract}
First, the X-ray source populations of M31 and M33 as known from Einstein and ROSAT observations are presented. Then, Chandra results on the galaxies are shortly summarized which not only spatially resolved the centre areas but also supernova remnants (SNRs) in both galaxies, and led to source catalogues of restricted areas with high astrometric accuracy. Also luminosity function studies and studies of individual sources based on Chandra and XMMNewton observations led to a better knowledge of the X-ray source populations. After that I will concentrate on XMM-Newton surveys, in which more than 850 and $400 \mathrm{X}$-ray sources were detected in M31 and M33, respectively, down to a $0.2-4.5 \mathrm{keV}$ luminosity of less than $10^{35} \mathrm{erg} \mathrm{s}^{-1}$. EPIC hardness ratios as well as informations from earlier X-ray, optical, and radio catalogues were used to distinguish between different source classes (SNRs, supersoft sources (SSSs), X-ray binaries (XRBs), globular cluster sources within the galaxies, and foreground stars, and objects in the background). However, many sources could only be classified as hard.They may either be XRBs or Crab-like SNRs in the galaxies or background sources. Within M31, two globular cluster sources could be identified as low mass XRBs as they showed type I X-ray bursts. Many of the SSSs in both galaxies were identified as optical novae. Due to the high frequency of outbursts in the bulge area of M31 many novae can be monitored at the same time which makes the investigation of class properties much easier compared to novae in the Milky Way or the Magellanic Clouds.
\end{abstract}

Keywords. galaxies: individual (M31, M33), novae, cataclysmic variables, supernova remnants, $\mathrm{X}$-rays: galaxies, X-rays: binaries, X-rays: bursts

\section{Introduction}

The Andromeda galaxy M31 is a massive early-type galaxy similar to the Milky Way with a large stellar bulge which is located in the Local Group at a distance of $780 \mathrm{kpc}$ (Holland 1998; Stanek \& Garnavich 1998; i.e. 1" corresponds to 3.8 pc) and is seen under an inclination of $78^{\circ}$. The optical extent of the SA(s)b galaxy can be approximated by an inclination-corrected $D_{25}$ ellipse with a large diameter of 153.3' and axis ratio of 3.09 (de Vaucouleurs, de Vaucouleurs, Corwin et al. 1991; Tully 1998). In contrast to M31 and the Milky Way, M33 is a late-type spiral galaxy, type Sc, and does not have a stellar bulge. It is the third largest galaxy in the Local Group located at a distance of $795 \mathrm{kpc}$ (van den Bergh 1991), is seen under a relatively low inclination of $56^{\circ}$ (Zaritsky, Elston \& Hill 1989) and the optical extent can be approximated by an inclination-corrected $\mathrm{D}_{25}$ ellipse with large diameter of $64.4^{\prime}$ and axes ratio of 1.66. With their moderate Galactic foreground absorption $\left(N_{\mathrm{H}}=6-7 \times 10^{20} \mathrm{~cm}^{-2}\right.$; Stark, Gammie, Wilson, et al. 1992) both galaxies are well suited to study the X-ray source population and diffuse emission. M31 and M33 have been the target of most imaging X-ray missions. In the following I will discuss results from the Einstein, ROSAT, Chandra, and XMM-Newton observatories. 


\section{Einstein and ROSAT observations of M31 and M33}

In the M31 field, the Einstein X-ray observatory detected 108 individual X-ray sources brighter than $\sim 5 \times 10^{36} \mathrm{erg} \mathrm{s}^{-1}, 16$ of which were found to vary between Einstein observations (van Speybroeck, Epstein, Forman, et al. 1979; Collura, Reale \& Peres 1990; Trinchieri \& Fabbiano 1991). The sources were identified with young stellar associations, globular clusters (i.e. LMXBs) and SNRs (see e.g. Blair, Kirshner \& Chevalier 1981; Crampton, Hutchings, Cowley, et al. 1984). With the ROSAT HRI, Primini, Forman \& Jones (1993) reported 86 sources brighter than $\sim 10^{36} \mathrm{erg} \mathrm{s}^{-1}$ in the central area of M31, nearly half of which were found to vary when compared to previous Einstein observations. With a separation of about one year between the M31 surveys, the ROSAT PSPC covered the entire galaxy twice and detected 560 X-ray sources down to a limit of $\sim 5 \times 10^{35} \mathrm{erg} \mathrm{s}^{-1}$ (Supper, Hasinger, Pietsch, et al. 1997; Supper, Hasinger, Lewin, et al. 2001). The intensity of 34 of the sources varied significantly between the observations, and SSSs were established as a new class of M31 X-ray sources (see also Kahabka 1999). Many of the bright sources were identified as globular cluster sources.

In the M33 field, the Einstein X-ray Observatory detected diffuse emission from hot gas and 17 unresolved sources (Long, Dodorico, Charles, et al. 1981; Markert \& Rallis 1983; Trinchieri, Fabbiano \& Peres 1988). First ROSAT HRI and PSPC observations revealed 57 sources and confirmed the detection of diffuse X-ray emission which may trace the spiral arms within a 10' radius around the nucleus (Schulman \& Bregman 1995; Long, Charles, Blair, et al. 1996). Combining all archival ROSAT observations of the field, Haberl \& Pietsch (2001) found 184 X-ray sources within a 50' radius around the nucleus, identified some of the sources by correlations with previous X-ray, optical and radio catalogues, and in addition classified sources according to their X-ray properties. They found candidates for SSSs, XRBs, SNRs, foreground stars, and active galactic nuclei (AGN).

Two M33 sources are known for their outstanding X-ray properties (Peres, Reale, Collura, et al. 1989). The brightest source (X-8 in the nomenclature of Long, Dodorico, Charles, et al. (1981), luminosity of $10^{39-40} \mathrm{erg} \mathrm{s}^{-1}$ ) is the most luminous X-ray source in the Local Group of galaxies and coincides with the optical center of M33. Its time variability (Dubus, Charles, Long, et al. 1997) and its point-like nature seen by ROSAT HRI (Pietsch \& Haberl 2000) point towards a black hole XRB. A possible periodicity of 106 days was not confirmed in later observations (see Parmar, Sidoli, Oosterbroek, et al. 2001). The second source $(\mathrm{X}-7)$ is an eclipsing high mass XRB (HMXB) with a binary period of $3.45 \mathrm{~d}$ and possible $0.31 \mathrm{~s}$ pulsations, discovered in ROSAT PSPC and HRI observations (Schulman, Bregman, Collura, et al. 1993; Dubus, Charles, Long, et al. 1997; Dubus, Charles, Long, et al. 1999; Larson \& Schulman 1997).

\section{Chandra and XMM-Newton observations of M31 and M33}

With the new generation of X-ray observatories, Chandra and XMM-Newton, only parts of M31 were surveyed. Chandra ACIS-I and HRC observations of the central region (covering areas of 0.08 and 0.27 square degree) resolved 204 and 142 X-ray sources, respectively (Garcia, Murray, Primini, et al. 2000; Kong, Garcia, Primini, et al. 2002a; Kaaret 2002). Three M31 disk fields, spanning a range of stellar populations, were covered with short Chandra ACIS-S observations to compare their point source luminosity functions to that of the galaxy's bulge (Kong, DiStefano, Garcia, et al. 2003a). A synoptic study of M31 with the Chandra HRC covered "most" of the disk (0.9 square degree) in 17 epochs using short observations, and resulted in mean fluxes and long-term light 
curves for the 166 objects detected (Williams, Garcia, Kong, et al. 2004). In these observations, several M31 SNRs were spatially resolved (Kong, Garcia, Primini, et al. 2002b; Kong, Sjouwerman, Williams, et al. 2003b: Williams, Sjouwerman, Kong, et al. 2004) and bright XRBs in globular clusters and SSSs and quasisoft sources (QSSs) could be characterized (Di Stefano, Kong, Garcia, et al. 2002; Di Stefano, Kong, Greiner, et al. 2004; Greiner, Di Stefano, Kong, et al. 2004). During the XMM-Newton guaranteed time program there were four observations of the central area of M31 and three aimed at the northern and two at the southern disk. These observations were used to investigate the bright and variable sources and diffuse emission (Shirey, Soria, Borozdin, et al. 2001; Osborne, Borozdin, Trudolyubov, et al. 2001; Trudolyubov, Borozdin \& Priedhorsky 2001; Trudolyubov, Kotov, Priedhorsky, et al. 2004), and to derive source luminosity distributions (Trudolyubov, Borozdin, Priedhorsky, et al. 2002a). In addition, the time variability and spectra of several individual XRBs have been studied in detail (e.g. Trudolyubov, Borozdin, Priedhorsky, et al. 2002b; Barnard, Kolb \& Osborne 2003; Barnard, Osborne, Kolb, et al. 2003; Barnard, Kolb \& Osborne 2004; Mangano, Israel \& Stella 2004; Barnard, Foulkes, Haswell, et al. 2005).

The full extent of M33 was covered by a homogeneous XMM-Newton survey with a sensitivity of $10^{35} \mathrm{erg} \mathrm{s}^{-1}$ in the $0.5-10 \mathrm{keV}$ band, a factor of ten deeper than previous surveys. The survey consisted of a raster of 15 pointings of about $10 \mathrm{ks}$ each. The directions were selected in a way that each area within the M33 optical $\mathrm{D}_{25}$ extent was covered by the XMM-Newton EPIC detectors at least three times with the medium filter. First results of this survey were presented in Pietsch, Ehle, Haberl, et al. (2003). Three Chandra ACIS-S and ACIS-I observations were pointed at the M33 nucleus and the starforming region NGC 604. Grimm, McDowell, Zezas, et al. (2005) present a source list of 261 sources in an area of $\sim 0.2$ square degree down to a luminosity of $\sim 2 \times 10^{34} \mathrm{erg} \mathrm{s}^{-1}$. They determine luminosity functions consistens with those of other star-forming galaxies. Based on the same observations, Ghavamian, Blair, Long, et al. (2005) report the detection of 22 optically known SNRs in M33 of which at least four ar spacially extended, and propose the identification of one non-radiative SNR (based on the spectral index of the radio counterpart) and new optical counterparts for two soft X-ray SNR candidates from the XMM-Newton list of Pietsch, Misanovic, Haberl, et al. (2004).

Chandra observations confirm the point-like nature of M33 X-8 (Dubus \& Rutledge 2002). The X-ray spectrum of the source can best be described by an absorbed power law plus disk blackbody model and the source flux can double within $5 \mathrm{ks}$ (e.g. Ehle, Pietsch \& Haberl 2001; La Parola, Damiani, Fabbiano, et al. 2003; Foschini, Rodriguez, Fuchs, et al. 2004; Dubus, Charles \& Long 2004). The combination of the properties establishes the source as the nearest example of the ultra-luminous X-ray sources that have been uncovered in other nearby galaxies.

Pietsch, Mochejska, Misanovic, et al. (2004) analyzed XMM-Newton and Chandra data of M33 X-7 and determined improved binary parameters, however, they could not confirm the proposed pulsations. In a special analysis of DIRECT data, they identified an O7I star of $18.89 \mathrm{mag}$ in $\mathrm{V}$, as the optical counterpart, which shows the ellipsoidal heating light curve of a HMXB with the $\mathrm{X}-7$ binary ephemeris. $\mathrm{X}-7$ was the most distant eclipsing XRB until the detection of another such source in XMM-Newton and Chandra observations of the starburst galaxy NGC 253, which is located at more than three times the distance of M33 (Pietsch, Haberl \& Vogler 2003).

\section{XMM-Newton surveys of M31 and M33}

Pietsch, Misanovic, Haberl, et al. (2004) have created merged medium and thin filter images for the three EPIC instruments, in five energy bands $(0.2-0.5 \mathrm{keV}, 0.5-1.0 \mathrm{keV}$, 

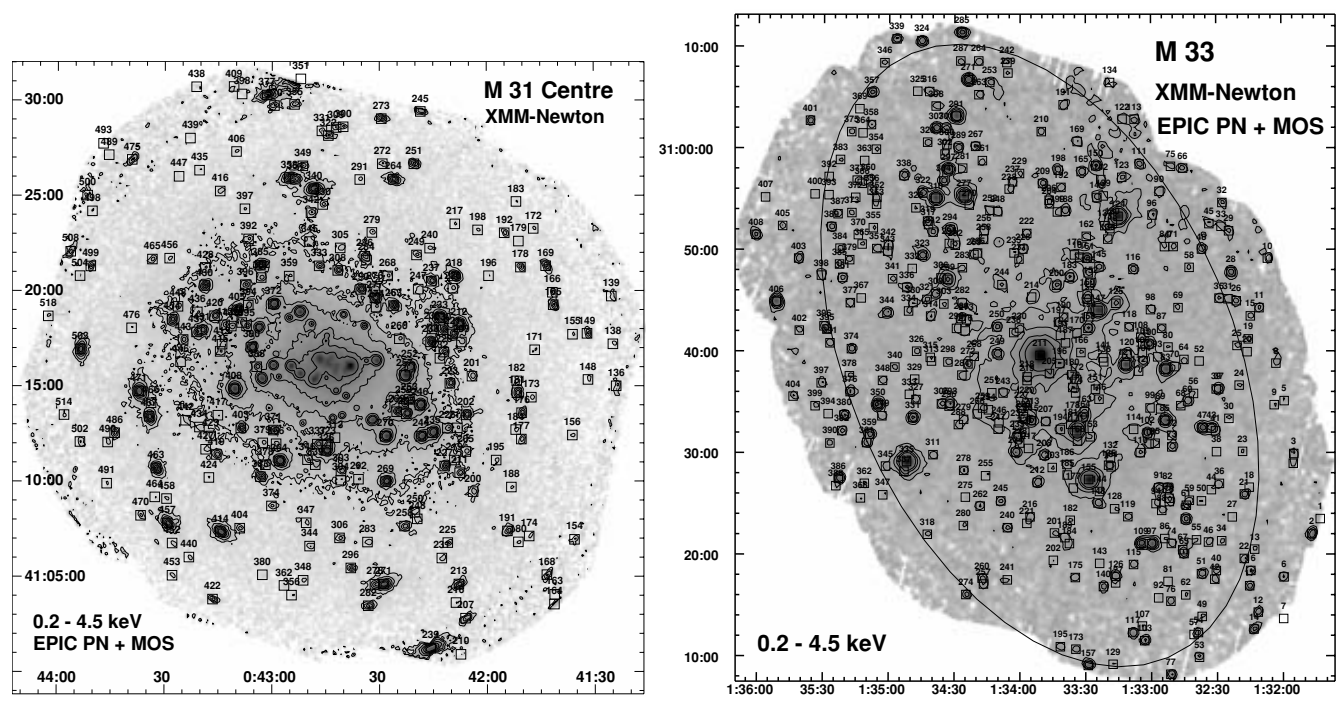

Figure 1. Combined XMM-Newton EPIC images in the $0.2-4.5 \mathrm{keV}$ band. (left): M31 centre observations smoothed with a 5" FWHM Gaussian (Fig. 1 from Pietsch, Freyberg \& Haberl (2005)). (right): M33 raster observations smoothed with a 20" FWHM Gaussian (Fig. 2 from Pietsch, Misanovic, Haberl, et al. (2004)).

1.0-2.0 keV, 2.0-4.5 keV, and 4.5-12 keV), using only times of low background from the XMM-Newton survey of M33†. A source search in these merged images - simultaneously using $5 \times 3$ images ( 5 energy bands and PN, MOS1 and MOS2 camera) - yielded a catalogue of 408 sources in an area of 0.80 square degree down to a luminosity in the $0.2-4.5 \mathrm{keV}$ band of $10^{35} \mathrm{erg} \mathrm{s}^{-1}$, more than a factor of 10 deeper than earlier ROSAT observations (see Fig. 1 for an overlay of the sources on a smoothed $0.2-4.5 \mathrm{keV}$ all EPIC image). Hardness ratios were calculated only for sources for which at least one of the two band count rates had a significance greater than $2 \sigma$. In search for identifications, the Xray source positions were correlated with sources in the SIMBAD and NED archives and within several catalogues. The catalogued X-ray sources are "identified" or "classified" based on properties in X-rays (hardness ratios (HR), variability, extent) and of correlated objects in other wavelength regimes. A source is counted as identified, if at least two criteria secure the identification. Otherwise, it is only counted as classified.

For the source catalogue and source population study of the archival observations of M31, Pietsch, Freyberg \& Haberl (2005) analyzed the individual pointings and the merged data of the central area simultaneously in five energy bands in a similar way as described in their M33 analysis. The covered area of 1.24 square degree and limiting sensitivity is a significant improvement compared to the Chandra surveys, but only covers about $2 / 3$ of the optical M31 extent ( $D_{25}$ ellipse) with a rather inhomogeneous exposure. The left panel of Fig. 1 shows an overlay of detected sources on an smoothed image of the M31 centre area. Fig. 2 shows colour/colour diagrams based on the HRs. To identify areas of specific source classes in the plots, colours of sources are over-plotted, that were derived from measured XMM-Newton spectra and model simulations.

$\dagger$ An analysis of the individual M33 pointings with less stringent background rejection and using all filters is presented in two posters by Misanovic et al. at this conference, and resulted in a source catalogue with improved positions (taking care of individual pointing offsets), that also provide information on source variability and spectral characteristics. 

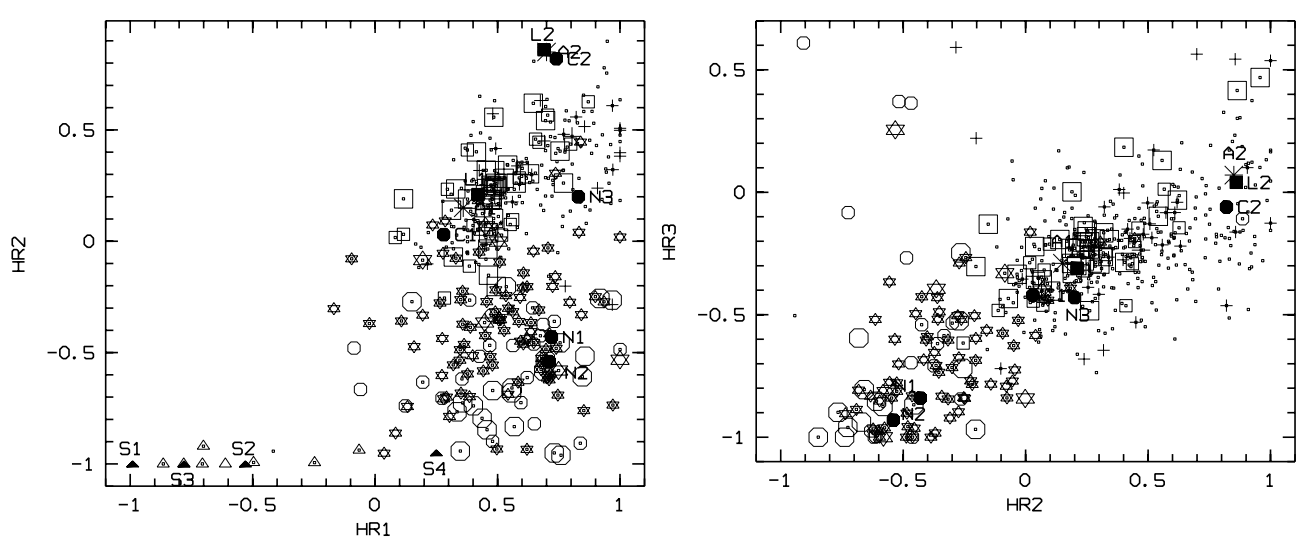

Figure 2. Hardness ratios detected by XMM-Newton EPIC. Shown as dots are only sources with HR errors smaller than 0.2 on both $H R i$ and $H R i+1$. Foreground stars and candidates are marked as big and small stars, AGN and candidates as big and small crosses, SSS candidates as triangles, SNR and candidates as big and small hexagons, GlCs and XRBs and candidates as big and small squares. In addition, we mark positions derived from measured XMM-Newton EPIC spectra and models for SSSs (S1 to S4) as filled triangles, low mass XRBs (L1 and L2) as filled squares, SNRs (N132D as N1, 1E 0102.2-7219 as N2, N157B as N3, Crab spectra as C1 and C2) as filled hexagons, AGN (A1 and A2) as asterisk. (Extracted from Fig. 5 from Pietsch, Freyberg \& Haberl (2005).)

Table 1. Summary of identifications and classifications of X-ray sources in M31 and M33.

\begin{tabular}{|c|c|c|c|c|c|c|c|c|c|c|}
\hline & & fgStar & AGN & GAL & $\mathrm{GAL} \mathrm{Cl}$ & SSS & SNR & $\mathrm{GlC}$ & $\mathrm{XRB}$ & hard \\
\hline \multirow[t]{2}{*}{ M31 } & identified & 6 & 1 & 1 & 1 & & 21 & 27 & 7 & \\
\hline & classified & 90 & 36 & & 1 & 18 & 23 & 10 & 9 & 567 \\
\hline \multirow[t]{2}{*}{ M33 } & identified & 5 & & 1 & & & $21+2$ & & 2 & \\
\hline & classified & 30 & 12 & 1 & & 5 & $23-2$ & & & 267 \\
\hline
\end{tabular}

Table 1 summarizes identifications and classifications according to the XMM-Newton catalogues. For the SNRs in M33 the Ghavamian, Blair, Long, et al. 2005 identifications are indicated. Detection of strong time variability in follow up analysis will certainly move many objects from the "hard" to "XRB" classification.

Based on the catalogue identifications, Pietsch \& Haberl (2005) searched for X-ray bursts in XMM-Newton archival data of X-ray sources in M31 globular clusters (GCs) and GC candidates. Two bursts were detected simultaneously in EPIC pn and MOS detectors and some more candidates in EPIC pn. The energy distribution of the burst photons and the intrinsic luminosity during the peak of the bursts indicate that at least the strongest burst was a type I radius expansion burst (Fig. 3). The bursts identify the sources as neutron star low mass X-ray binaries in M31. The type I X-ray bursts in M31 are the first detected outside the Milky Way and show that with the help of XMM-Newton X-ray bursts can be used to classify neutron star low mass X-ray binaries in Local Group galaxies.

\section{Novae as major class of SSS in M31}

Pietsch, Fliri, Freyberg, et al. (2005) searched for X-ray counterparts to optical novae detected in M31 and M33. They combined an optical nova catalogue from the WeCAPP survey with optical novae reported in the literature and correlated them with the most 

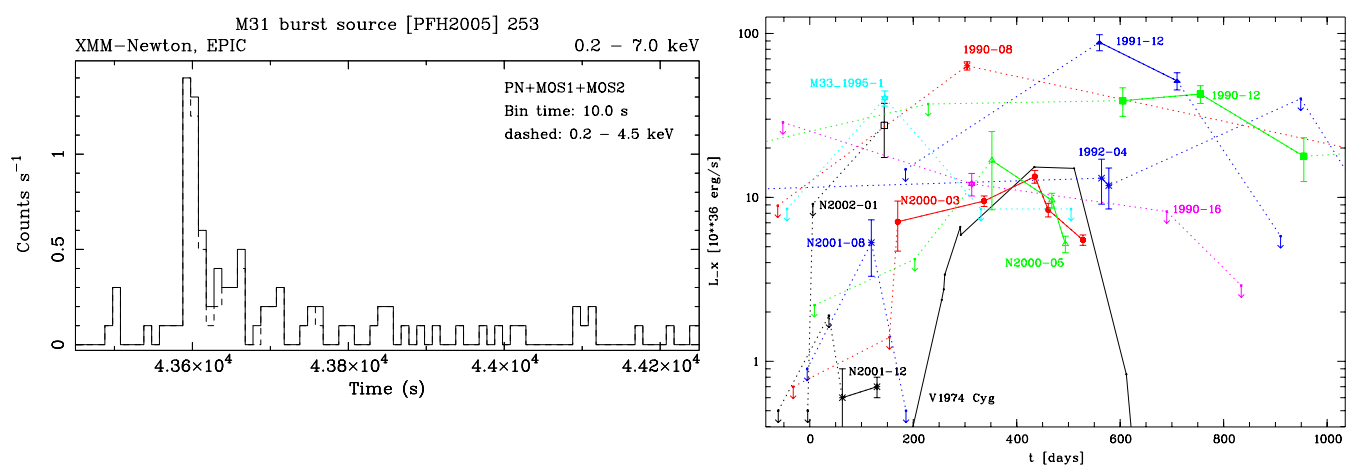

Figure 3. (left): Combinded XMM-Newton EPIC light curve of a type I X-ray burst of source [PFH2005] 253 in M31 (Fig. 3 from Pietsch \& Haberl (2005)). (right): Light curves for M31 and M33 novae that were detected within $1000 \mathrm{~d}$ after outburst. Detections of individual novae are connected by solid lines, and connections to upper limits are marked by dashed lines (Fig. 3 from Pietsch, Fliri, Freyberg, et al. (2005)).

recent X-ray catalogues from ROSAT, XMM-Newton and Chandra, and - in addition searched for nova correlations in archival data. They report $21 \mathrm{X}$-ray counterparts for novae in M31 (mostly SSS) and two in M33. This sample more than triples the number of known optical novae with supersoft phase. Most of the counterparts are covered in several observations which allows to constrain their X-ray light curves (see Fig. 3). Selected brighter sources were classified by their XMM-Newton EPIC spectra. Six counterparts are only detected in Chandra HRC I (3) or ROSAT HRI (3) observations, i.e. X-ray detectors with no energy resolution, and therefore can not be classified as supersoft. From the well-determined start time of the SSS state in two novae one can estimate the hydrogen mass ejected in the outburst to $\sim 10^{-5} M_{\odot}$ and $\sim 10^{-6} M_{\odot}$, respectively. The supersoft X-ray phase of at least $15 \%$ of the novae starts within a year. At least one of the novae shows a SSS state lasting 6.1 years after the optical outburst. Six of the SSSs turned on between 3 and 9 years after the optical discovery of the outburst and may be interpreted as recurrent novae. If confirmed, the detection of a delayed SSS phase turn-on may be used as a new method to classify novae as recurrent. At the moment, the new method yields a ratio of recurrent novae to classical novae of 0.3 .

\section{Conclusions}

The sensitivity of XMM-Newton and Chandra observations of M31 and M33 combined with the wealth of multi-wavelength data for these galaxies allows a detailed study of the point source population. Many more interesting results can be expected from the approved Chandra ACIS-I very large proposal on M33 (sensitive mainly above $0.5 \mathrm{keV}$ ) and, hopefully, further monitoring of M31 and M33 with XMM-Newton and Chandra also in the energy band below $0.5 \mathrm{keV}$. The first results of the monitoring the SSS state of optical novae proved that these kind of studies can be more efficiently achieved by observing many candidates at the same time in one field in M31 or M33 than by monitoring individual sources in the Milky Way or the Magellanic Clouds. The results of the Chandra and XMM-Newton observations of M31 and M33 demonstrate the importance of arcsec spatial resolution, broad energy coverage, good energy resolution, and high collecting power - used together with deep images and catalogues at other wavelengths also for future X-ray source population studies in nearby galaxies. 


\section{References}

Barnard, R., Osborne, J. P., Kolb, U., \& Borozdin, K. N. 2003, A $\& A$ 405, 505

Barnard, R., Kolb, U., \& Osborne, J. P. 2003, A\& A 411, 553

Barnard, R., Kolb, U., \& Osborne, J. P. 2004, A\&A A 423, 147

Barnard, R., Foulkes, S. B., Haswell, C. A., Kolb, U., Osborne, J. P., \& Murray, J. R. 2005, MNRAS submitted (astro-ph/0503259)

Blair, W. P., Kirshner, R. P., \& Chevalier, R. A. 1981, ApJ 247, 879

Collura, A., Reale, F., \& Peres, G. 1990, ApJ 356, 119

Crampton, D., Hutchings, J. B., Cowley, A. P., Schade, D. J., \& van Speybroeck, L. P. 1984, ApJ 284, 663

de Vaucouleurs, G., de Vaucouleurs, A., Corwin, H. G., Buta, R. J., Paturel, G., \& Fouque, P. 1991, Third Reference Catalogue of Bright Galaxies (vol. 1-3, Springer-Verlag Berlin Heidelberg New York)

Di Stefano, R., Kong, A. K. H., Garcia, M. R., Barmby, P., Greiner, J., Murray, S. S., \& Primini, F. A. 2002, ApJ 570, 618

Di Stefano, R., Kong, A. K. H., Greiner, J., Primini, F. A., Garcia, M. R., Barmby, P., Massey, P., Hodge, P. W., Williams, B. F., Murray, S. S., Curry, S., Russo, T. A., et al. 2004, ApJ 610,247

Dubus, G., Charles, P. A., Long, K. S., \& Hakala, P. J. 1997, ApJ (Letters) 490, L47

Dubus, G., Charles, P. A., Long, K. S., Hakala, P. J., \& Kuulkers, E. 1999, MNRAS 302, 731

Dubus, G. \& Rutledge, R. E. 2002, MNRAS 336, 901

Dubus, G., Charles, P. A., \& Long, K. S. 2004, A\& $A$ 425, 95

Ehle, M., Pietsch, W., \& Haberl, F. 2001, in ASP Conf. Ser. 251: New Century of X-ray Astronomy, 300

Foschini, L., Rodriguez, J., Fuchs, Y., Ho, L. C., Dadina, M., Di Cocco, G., Courvoisier, T. J.-L., \& Malaguti, G. 2004, A\&̈A 416, 529

Garcia, M. R., Murray, S. S., Primini, F. A., Forman, W. R., McClintock, J. E., \& Jones, C. 2000, ApJ (Letters) 537, L23

Ghavamian, P., Blair, W. P., Long, K. S., Sasaki, M., Gaetz, T. J., \& Plucinsky, P. P. 2005, AJ 130,539

Greiner, J., Di Stefano, R., Kong, A., \& Primini, F. 2004, ApJ 610, 261

Grimm, H.-J., McDowell, J., Zezas, A., Kim, D.-W., \& Fabbiano, G. 2005, ApJS accepted (astro-ph/0506353)

Haberl, F. \& Pietsch, W. 2001, A\&SA 373, 438 [HP01]

Holland, S. 1998, AJ 115, 1916

Kaaret, P. 2002, textitApJ 578, 114

Kahabka, P. 1999, A\&A 344, 459

Kong, A. K. H., Garcia, M. R., Primini, F. A., Murray, S. S., Di Stefano, R., \& McClintock, J. E. 2002a, textitApJ 577, 738

Kong, A. K. H., Garcia, M. R., Primini, F. A., \& Murray, S. S. 2002b, ApJ (Letters) 580, L125

Kong, A. K. H., DiStefano, R., Garcia, M. R., \& Greiner, J. 2003a, ApJ 585, 298

Kong, A. K. H., Sjouwerman, L. O., Williams, B. F., Garcia, M. R., \& Dickel, J. R. 2003b, ApJ (Letters) 590, L21

La Parola, V., Damiani, F., Fabbiano, G., \& Peres, G. 2003, ApJ 583, 758

Larson, D. T. \& Schulman, E. 1997, AJ 113, 618

Long, K. S., Dodorico, S., Charles, P. A., \& Dopita, M. A. 1981, ApJ (Letters) 246, L61

Long, K. S., Charles, P. A., Blair, W. P., \& Gordon, S. M. 1996, ApJ 466, 750

Mangano, V., Israel, G. L., \& Stella, L. 2004, A\&A 419, 1045

Markert, T. H. \& Rallis, A. D. 1983, ApJ 275, 571

Osborne, J. P., Borozdin, K. N., Trudolyubov, S. P., Priedhorsky, W. C., Soria, R., Shirey, R., Hayter, C., La Palombara, N., Mason, K., Molendi, S., Paerels, F., Pietsch, W., Read, A. M., Tiengo, A., Watson, M. G., \& West, R. G. 2001, A\& $A$ 378, 800

Parmar, A. N., Sidoli, L., Oosterbroek, T., Charles, P. A., Dubus, G., Guainazzi, M., Hakala, P., Pietsch, W., \& Trinchieri, G. 2001, A\&A 368, 420

Peres, G., Reale, F., Collura, A., \& Fabbiano, G. 1989, ApJ 336, 140 
Pietsch, W. \& Haberl, F. 2000, in The interstellar medium in M31 and M33. Proceedings 232. WE-Heraeus Seminar, 22-25 May 2000, Bad Honnef, Germany. Edited by Elly M. Berkhuijsen, Rainer Beck \& Rene A. M. Walterbos. Shaker, Aachen, 2000, p. 149

Pietsch, W., Haberl, F., \& Vogler, A. 2003, A $\mathscr{H} A$ 402, 457

Pietsch, W., Ehle, M., Haberl, F., Misanovic, Z., \& Trinchieri, G. 2003, Astronomische Nachrichten 324,85

Pietsch, W., Mochejska, B. J., Misanovic, Z., Haberl, F., Ehle, M., \& Trinchieri, G. 2004, A\&A 413,879

Pietsch, W., Misanovic, Z., Haberl, F., Hatzidimitriou, D., Ehle, M., \& Trinchieri, G. 2004, A\& A 426,11

Pietsch, W. \& Haberl, F. 2005, A\&A 430, L45

Pietsch, W., Freyberg, M., \& Haberl, F. 2005, A\&A 434, 483

Pietsch, W., Fliri, J., Freyberg, M. J., Greiner, J., Haberl, F., Riffeser, A., \& Sala, G. 2005, $A \mathscr{E} A$ in press (astro-ph/0504321)

Primini, F. A., Forman, W., \& Jones, C. 1993, ApJ 410, 615

Schulman, E., Bregman, J. N., Collura, A., Reale, F., \& Peres, G. 1993, ApJ (Letters) 418, L67

Schulman, E. \& Bregman, J. N. 1995, ApJ 441, 568

Shirey, R., Soria, R., Borozdin, K., Osborne, J. P., Tiengo, A., Guainazzi, M., Hayter, C., La Palombara, N., Mason, K., Molendi, S., Paerels, F., Pietsch, W., Priedhorsky, W., Read, A. M., Watson, M. G., \& West, R. G. 2001, A\& $A$ 365, L195

Stanek, K. Z. \& Garnavich, P. M. 1998, ApJ (Letters) 503, L131

Stark, A. A., Gammie, C. F., Wilson, R. W., Bally, J., Linke, R. A., Heiles, C., \& Hurwitz, M. 1992, ApJS 79, 77

Supper, R., Hasinger, G., Lewin, W. H. G., Magnier, E. A., van Paradijs, J., Pietsch, W., Read, A. M., \& Trümper, J. 2001, A\&\&A 373, 63

Supper, R., Hasinger, G., Pietsch, W., Trümper, J., Jain, A., Magnier, E. A., Lewin, W. H. G., \& van Paradijs, J. 1997, A\& A 317, 328

Trinchieri, G., Fabbiano, G., \& Peres, G. 1988, ApJ 329, 1037

Trinchieri, G. \& Fabbiano, G. 1991, ApJ 382, 82

Trudolyubov, S. P., Borozdin, K. N., \& Priedhorsky, W. C. 2001, ApJ (Letters) 563, L119

Trudolyubov, S. P., Borozdin, K. N., Priedhorsky, W. C., Mason, K. O., \& Cordova, F. A. 2002a, ApJ (Letters) 571, L17

Trudolyubov, S. P., Borozdin, K. N., Priedhorsky, W. C., Osborne, J. P., Watson, M. G., Mason, K. O., \& Cordova, F. A. 2002b, ApJ (Letters) 581, L27

Trudolyubov, S., Kotov, O., Priedhorsky, W., Cordova, F., \& Mason, K. 2004, ApJ submitted (astro-ph/0401227)

Tully, R. B. 1988, Nearby galaxies catalog (Cambridge and New York, Cambridge University Press)

van den Bergh, S. 1991, PASP 103, 609

van Speybroeck, L., Epstein, A., Forman, W., Giacconi, R., Jones, C., Liller, W., \& Smarr, L. 1979, ApJ (Letters) 234, L45

Williams, B. F., Garcia, M. R., Kong, A. K. H., Primini, F. A., King, A. R., Di Stefano, R., \& Murray, S. S. 2004, ApJ 609, 735

Williams, B. F., Sjouwerman, L. O., Kong, A. K. H., Gelfand, J. D., Garcia, M. R., \& Murray, S. S. $2004, A p J 615,720$

Zaritsky, D., Elston, R., \& Hill, J. M. 1989, AJ 97, 97 


\section{Discussion}

ERACLEOUS: Did you get any useful information from the optical monitor that can help with the source identification?

PIETsCH: Not all fields have OM observations. We have not looked into that yet, but in principle the UV information can help.

KIM: How many background AGNs do you expect in M31, M33 field of view and only in the bulge where the majority of the hard X-ray sources would be LMXBs? Did you make the luminosity function and compare with the Chandra results?

PIETSCH: In M33, about half of the detected sources are expected to be background AGN. In M31, we did not make an estimate. However, as the observations are more concentrated to the denser areas of the galaxy, the fraction should be less, specifically in the bulge area. We did not create luminosity functions yet.

DiStefano: This is a comment on the connection you have discussed between SSSs and novae. Among SSSs in the Galaxy and Magellanic Clouds (the so-called "classical" SSSs), novae comprise only a small fraction of SSSs. In more distant spirals, such as M101, M83, and M51 a large fraction of SSSs are found in the spiral arms, not consistent with a population that is previously old. This is true for even the softest sources in the galaxies.

PIETSCH: I made clear in the talk that if not all but most of the SSS in M31 correlate with novae. Many of the QSS in more distant galaxies are not SSS in the strong spectral selection. However, even if they were SSS, it would be difficult to identify with optical novae as there is no nova monitoring in these galaxies. 